\title{
Analysis of Pointing Tasks on a White Board*
}

\author{
G. Faconti and Mieke Massink \\ Consiglio Nazionale delle Ricerche, Istituto ISTI, Pisa, Italy \\ \{G.Faconti, M.Massink\}@isti.cnr.it
}

\begin{abstract}
We study the variations in two dimensional (2D) pointing tasks on a traditional white board of a group of subjects by means of capturing their movement traces in an automatic way with the Mimio device. Such traces provide detailed insight in the variability of $2 \mathrm{D}$ pointing relevant for example for the design of computer vision based gestural interaction. This study provides experimental evidence that for medium large distances Fitts' model, and Welfords and Shannons variants, continue to show a linear relationship between movement time (MT) and the index of difficulty (ID) with a high correlation for the ranges considered. The expected increased sensitivity to changes in ID for these larger distances are confirmed. Nearly all movements show three phases: a planning phase, a ballistic phase and an adjustment phase. Finally, we show that the arrival time at the target resembles a log-normal distribution.
\end{abstract}

\section{Introduction}

One of the challenges in Human-Computer Interaction is to let computers support activities that humans already perform in their daily life with the tools and environment they are used to work with. The computer support to such activities should ideally interfere as little as possible with the human activities but nevertheless provide a real augmented reality.

However, in order to provide real-time and adequate support to the user, the computing system needs to operate in a tightly coupled, continuous way with the activities of the user and its environment. The increase of computing power, the miniaturisation and the enormous developments in devices for data-acquisition such as video cameras and related image analysis software have stimulated much research and experimentation with computer vision based gestural interaction techniques for human-computer interaction 34.

Although computer vision based techniques potentially enable a direct and continuous interaction between user and computer, the tight coupling requires that the software is able to keep up with user's movements. This is a challenging enterprise in particular due to the variability of human behaviour even in simple and repetitive tasks. Systems that do not manage to keep up sufficiently close or behave unpredictably may constrain the user's activities and disturb or even

\footnotetext{
* Research partially funded by EU Integrated Project Sensoria, contract n. 016004.
} 
interrupt higher-level cognitive activities that are performed in parallel with the pointing behaviour [2].

One kind of application that has received considerable attention in the literature is that of finger tracking. Finger tracking is a computer vision technique that allows a computer in combination with a video camera to follow finger movements of users when they are working, for example, with a white board. Used in combination with a projector, finger tracking can be used as part of an augmented reality application for the white board. In such a setting the user uses a mix of common physical and virtual devices such as pens and erasers for the white board and projected virtual buttons for operations such as copy and paste. A nice experimental example of such a device is the Magic Board [311.

Experimental design of the Magic Board required an investigation of the velocity of the natural pointing movements that people perform when using a white board. At the time of its design experiments were performed to estimate such velocity using a video camera that captured the position of the user's finger with a frequency of 25 images per second. These data have been analysed image per image in a non-automatised way $[3$.

There exist also well-known and useful models, such as Fitts' law and its many variants that provides us with an estimate of movement times based on an index of difficulty and an index of performance. However, such models typically provide only mean movement times and for distances of at most $40 \mathrm{~cm}$. which involve usually smaller limb groups, such as fingers, wrist and forearm, than those involved in $2 \mathrm{D}$ pointing on a white board. These models have been mainly developed to predict the time to position a cursor or to select a target employing devices such as the mouse, a touch pad and numerous other devices that can be found in traditional desk-top computers [1013115]. Some studies suggest that movements that involve large limbs are more sensitive to changes in the index of difficulty 1012 .

Pointing in the context of a white board involves much larger distances over which we expected the velocity to vary considerably during single movements. In this paper we therefore revisit the finger pointing experiment for the white board, but instead of a video camera we use the Mimio [8], a high-resolution ultrasonic position capturing device that can be attached to a normal white board and which can be used to register automatically and in real-time the exact trajectory of a pen that is moved over the white board by the user. This allows for a much more detailed analysis than was possible with a video camera. We study the trajectories of adult subjects for simple pointing tasks on a white board. We investigate to what extend Fitts' model is appropriate for these larger movements. We also investigate the maximal velocity of the pen in relation to the distance from the target and the distribution of the time to reach the target for various distances. Such distributions provide information about the variability of the pointing behaviour, which is usually not completely random, but rather well-approximated by stochastic distributions. Such distributions in their turn are useful in stochastic models of combined user and system behaviour such as discussed in some of our earlier work [5]. 
Sect. 2 describes the design of the experiment and in Sect. 3 we analyse the obtained data. Sect. 4 presents a discussion. An extended version of this article is available as technical report [6].

\section{The White Board Pointing Experiment}

For the experiments we have asked 18 participants to perform a number of pointing tasks on a white board with the Mimio device. In this section we provide more details on the participants and the experimental set-up.

\subsection{Participants}

18 participants took part in the experiment (12 male and 6 female), aged between 17 and 54 years, with an average age of 40 years. They were students, Researchers and Professors at University, and teachers at High School. All of them were native Italian speakers with normal or adjusted to normal vision and right-handed.

\subsection{Apparatus}

For the experiments a Mimio capture bar device [8] was used for the measurement of positional data. The capture bar was positioned over a vertically mounted white board of $1050 \mathrm{~mm}$. high and $1400 \mathrm{~mm}$. wide, positioned at $1200 \mathrm{~mm}$. from the floor, as illustrated in Fig. 1. It was connected through a Universal Serial Bus (USB) port to a portable computer equipped with a Pentium III $850 \mathrm{MHz}$ processor and 128 Mbytes of memory. $x$ and $y$ co-ordinates of the position of the tip of a Mimio provided pen, held by the participant while sliding over the board, were recorded in $\mathrm{mm}$. and time-stamped at the computer. The pen resembles conventional markers used for writing on white boards. The difference is that in the Mimio pen the conventional marker is enclosed in a hard plastic wrapper which is slightly larger than that of the marker pen it contains carrying infrared and ultrasound transmitters. The device is ergonomically designed to be held and used by a person as if it was a traditional marker with slightly increased diameter. When the device is pushed against the board, as normally happens when writing, a micro-switch is operated and two signals (infrared and ultrasound) are generated that are sensed by the capture bar. When the device is released from the board, the micro-switch stops the generation of the signals. We have used the version of the pen that does not leave an ink-trace on the board in order not to distract the participants with already drawn lines while performing the experiment. The Mimio is able to determine the current position of the device relative to the board by triangulation with a resolution of $0.35 \mathrm{~mm}$. and a frequency of $87 \mathrm{~Hz}$.

\subsection{Stimuli}

Two sets of stimuli were presented marked on the board by means of circles of black ink. Both sets were formed by five circles with diameters measuring 
$20 \mathrm{~mm}$. and $10 \mathrm{~mm}$. respectively. We have opted for circles rather than squares, such as in other $2 \mathrm{D}$ pointing experiments [15], because of their invariance of the width to the angle of approach of the target. The targets were placed at the following positions (measurements are from the bottom-left corner of the white board): position 1 (150 mm. horizontal, $700 \mathrm{~mm}$. vertical); position 2 (950 mm., $700 \mathrm{~mm}$.$) ; Centre (600 mm., 450 \mathrm{~mm}$.$) ; position 3$ (150 mm., $200 \mathrm{~mm}$.$) ; and$ position 4 (950 mm., $200 \mathrm{~mm}$.) - see Fig. 1 for illustration. The positions reflect a reasonably representative set of pointing movements that are likely to occur when using a normal white board.
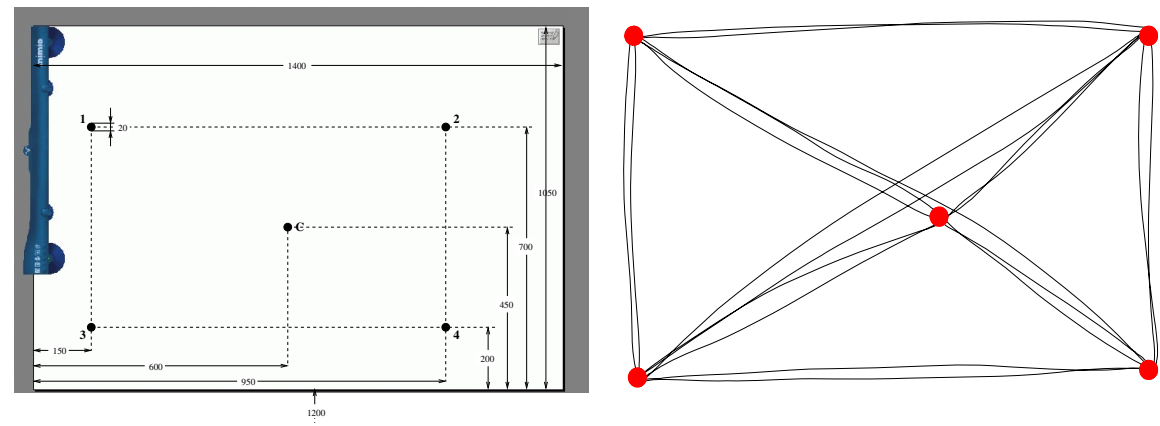

Fig. 1. Board with capture bar and position of stimuli (left) and trajectories of a single subject (right)

\subsection{Procedure}

The design of the experiment was that of a fully-crossed, within subjects factorial design with repeated measures. The participants were each provided with a Mimio pen for a practise trial before the beginning of the experiment. The trial was the same for all subjects and consisted of writing their name on the board.

The basic task of participants in the experiment was to connect two circles by pointing to the first with the pen, pushing it slightly on the board and sliding it to the second circle at a velocity that feels natural to the participant, according to verbal instructions. The sliding of the pen over the board is needed for the Mimio device to capture the trajectory. The five circles on the board gave 20 types of movement that a participant could be asked to perform. One response for each type of movement was obtained during the experiment. The movement types included both directions between the central circle and each of the four corner circles and also those between the four corner circles in the horizontal, vertical, and diagonal planes as shown in Fig. 11 on the right where the trajectories corresponding the 20 movements of a single subject are drawn. Tasks were presented in a random order for each participant. 
Participants were instructed as follows in Italian:

"On the board in front of you five circles are marked. The outer circles are numbered 1, 2, 3 and 4 (Experimenter demonstrates by pointing to each position). The central circle is simply denoted by the letter ' $\mathrm{C}$ '. Your task is to connect to circles with the pen you are holding according to my instructions. First I will tell you which circle to start at - I will say, for example, "From 1 ...". I will then tell you which circle you should move to - I will do this by giving you the label of the circle, for example "... to C". You should move your hand at a speed that feels natural to you sliding the pen across the board. Try to get the tip of the pen close to the centre of each target."

\section{Data Analysis}

The experimental data collected following the process described in the previous section were classified based on the distance of the two circles to be connected and the width of the target circle. Consequently, we identified (i) long diagonal movements (LD) from 1 to 4 , from 2 to 3 , and vice versa, (ii) middle diagonal movements (MD) from 1 and 3 to $\mathrm{C}$, and vice versa, (iii) short diagonal movements (SD) from 2 and 4 to $\mathrm{C}$, and vice versa, (iv) horizontal movements (HO) from 1 to 2 , from 3 to 4 , and vice versa, (v) vertical movements (VE) from 1 to 3 , from 2 to 4 , and vice versa (see Fig. 11). The five classes were replicated for the large and the small target respectively. This led to the identification of ten classes consisting of $18 \times 4$ measurements each. It might have been reasonable to split each class into two; one for each different direction of movement (i.e. left to right and right to left or downward and upward). However, given the rather small number of trials for each movement in this explorative study we decided to keep the above mentioned classes and examine the results for indications for the need for further refinement in future experiments.

\subsection{Fitts' Law Analysis of Overall Data}

Our analysis started from a consideration of Fitts' law [7] as one of the rare quantitative tools available in user interface research and development.

The length of movements studied in this experimental setting exceeds that usually considered in evaluating devices such as for example mice and tablets. As a consequence, the participants in our experiments need to use different limbs and muscles to perform the pointing tasks than in the usual Fitts' law experiments. The above observation justifies the potential for validating Fitts' formal relationship in the case of a white board equipped with a Mimio device although the main objective of our work aims at finding performance distributions and variations in pointing behaviour rather than the mean values of human perceptual-motor performance.

The usual form of Fitts' law predicts that the movement time $M T$ needed to point at a target of width $W$ at distance $D$ is logarithmically related to the inverse of the spatial relative error $\frac{2 D}{W}$, that is: 


$$
M T=a+b \log _{2}\left(\frac{2 D}{W}\right)
$$

where $a$ and $b$ are empirically determined constants [12].

There exist a number of well-known variations of Fitts' law such as Welford's variation 1819$]$ :

$$
M T=a+b \log _{2}\left(\frac{D}{W}+0.5\right)
$$

and Shannon's original theorem [16]:

$$
M T=a+b \log _{2}\left(\frac{D}{W}+1\right)
$$

The logarithmic factor in the formulas, called the index of difficulty $I D$, describes the difficulty to achieve the pointing task 12 . The index of performance $I P$, defined as $I P=\frac{I D}{(M T-a)}$, gives a measure of the information capacity of the human motor system, analogous to channel capacity $C$ in Shannon's theorem [16].

In our analysis we compared all above variants of Fitts' law. Also, we used the method described in [15] to compute the effective target size $W_{e}$ in a two dimensional space to replace $W$ in the above equations. The effective target size reflects the actual size of the target based on what the participants really did. Equation (3) with $W$ replaced by $W_{e}$ is also used in the new standard for pointing devices ISO9241-9 14. The use of $W_{e}$ instead of $W$ is believed to increase the accuracy of the model in general.

The overall results obtained from our experiments for all pointing tasks of all participants is given in Table 11. The table reports the results for the width of the targets considered $\left(20 \mathrm{~mm}\right.$. and $10 \mathrm{~mm}$.), the effective width $W_{e}$ and the distance $D$. The index of difficulty $I D$, the mean movement time $M T$ and the index of performance (or throughput) $I P$ have been calculated using $W_{e}$ for the three variants of Fitts' law: Fitts $\left(I D_{F}, I P_{F}\right)$, Welford $\left(I D_{W}, I P_{W}\right)$ and Shannon $\left(I D_{S}, I P_{S}\right)$. The last column in Table 1 gives the mean velocity for each combination of target and distance.

The results for Welfords variant are presented graphically in Fig. 2 together with a first order fit of the data to the logarithmic component of Welfords variant, the correlation coefficient of 0.98 , the regression coefficient of $0.675 \mathrm{~s} / \mathrm{bit}$ and its regression constant of -1.658 s. The results for Fitts' law and Shannon's variant are very similar with correlation coefficient 0.98 and 0.99 resp., regression coefficient $0.667 \mathrm{~s} /$ bit and $0.682 \mathrm{~s} /$ bit resp. and regression constant $-2.274 \mathrm{~s}$. and -1.711 s. resp. All results show a linear relationship between movement time and the index of difficulty with a high correlation as has also been observed in many other Fitts' law studies involving finger, wrist and forearm muscles in computer input control 1213. A difference with the results reported in 12] on Fitts' results for the tapping experiment involving distances of between 2 and 16 inches is the regression coefficient (slope). In Fitts' experiments the slope 
Table 1. Data from experiment with Mimio capture bar and pen

\begin{tabular}{|c|c|c|c|c|c|c|c|c|c|c|c|}
\hline $\begin{array}{c}W \\
(\mathrm{~mm})\end{array}$ & $\begin{array}{c}W_{e} \\
(\mathrm{~mm})\end{array}$ & $\begin{array}{c}D \\
(\mathrm{~mm})\end{array}$ & $\begin{array}{l}\text { Mov. } \\
\text { Type }\end{array}$ & $\begin{array}{l}I D_{e F} \\
\text { (Bits) }\end{array}$ & $\begin{array}{l}I D_{e W} \\
\text { (Bits) }\end{array}$ & $\begin{array}{l}I D_{e S} \\
\text { (Bits) }\end{array}$ & $\begin{array}{l}M T \\
(\mathrm{sec})\end{array}$ & $\begin{array}{c}I P_{e F}^{*} \\
(\mathrm{Bits} / \mathrm{s})\end{array}$ & $\begin{array}{c}I P_{e W}^{*} \\
(\mathrm{Bits} / \mathrm{s})\end{array}$ & $\begin{array}{c}I P_{e S}^{*} \\
(\mathrm{Bits} / \mathrm{s})\end{array}$ & $\begin{array}{c}\text { Velocity } \\
(\mathrm{cm} / \mathrm{s})\end{array}$ \\
\hline 20 & 17.98 & 430.1 & SD & 5.58 & 4.61 & 4.64 & 1.518 & 1.47 & 1.45 & 1.44 & 28.33 \\
\hline 20 & 18.21 & 500.0 & VE & 5.77 & 4.81 & 4.83 & 1.615 & 1.48 & 1.47 & 1.45 & 30.96 \\
\hline 20 & 18.27 & 514.9 & MD & 5.82 & 4.84 & 4.87 & 1.637 & 1.49 & 1.47 & 1.45 & 31.45 \\
\hline 20 & 18.35 & 800.0 & $\mathrm{HO}$ & 6.45 & 5.46 & 5.48 & 1.954 & 1.53 & 1.51 & 1.50 & 40.94 \\
\hline 20 & 19.12 & 943.4 & LD & 6.62 & 5.63 & 5.65 & 2.138 & 1.50 & 1.48 & 1.47 & 44.13 \\
\hline 10 & 7.83 & 430.1 & SD & 6.78 & 5.79 & 5.81 & 2.160 & 1.53 & 1.52 & 1.50 & 19.91 \\
\hline 10 & 8.25 & 500.0 & VE & 6.92 & 5.93 & 5.95 & 2.295 & 1.51 & 1.50 & 1.49 & 21.79 \\
\hline 10 & 8.07 & 514.9 & MD & 6.99 & 6.01 & 6.02 & 2.384 & 1.50 & 1.49 & 1.47 & 21.60 \\
\hline 10 & 8.28 & 800.0 & $\mathrm{HO}$ & 7.59 & 6.60 & 6.82 & 2.744 & 1.51 & 1.50 & 1.53 & 29.15 \\
\hline \multirow[t]{3}{*}{10} & 8.42 & 943.4 & LD & 7.81 & 6.81 & 6.82 & 3.097 & 1.45 & 1.43 & 1.42 & 30.46 \\
\hline & & & & & & Mean & 2.152 & 1.50 & $\overline{1.49}$ & 1.47 & \\
\hline & & & & & & StDev & 0.501 & 0.02 & 0.03 & 0.03 & \\
\hline
\end{tabular}

$(*)$ Calculated using $I P=I D /(M T-a)$ where $a$ is the regression constant.

for the experiment in which a stylus of $1 \mathrm{oz}$ was used is $0.1089 \mathrm{~s} / \mathrm{bit}$ and for the 1-lb stylus $0.1240 \mathrm{~s} /$ bit, which are both much lower than the regression coefficient found for the white board experiment. So, the index of difficulty has more influence on the movement time in the case of the white board than in the case of traditional desktop computer interfaces such as mouse and joy-stick. This result is in line with an hypothesis made in earlier research by Langolf et al. [10] in which it was found that IP decreased as the limb changed from the finger to the wrist to the forearm, i.e. involving increasingly larger limbs.

The mean velocity presented in Table 1 is much lower than the maximum velocity reported in [3]. In their experiments a maximum velocity of $200 \mathrm{~cm} / \mathrm{s}$ has been observed in pointing tasks where participants were asked to start from one extreme of the white board, i.e. covering approximately $120 \mathrm{~cm}$., and put a mark with a pen on the other extreme in a fast way. This shows, as expected, that the mean movement time and distance is not a satisfactory predictor of the maximal velocity that may occur in pointing movements over medium large distances.

In order to get better insight in the variation of the velocity during pointing tasks on the white board we analyse the obtained trajectories in the following sections.

\subsection{Convergence Patterns}

According to Jagacinski et al. 9] researchers have postulated in the past two classes of models that attempt to explain the movement processes underlying the relationship between target width and distance. One class postulates that the movement is composed of a sequence of discrete sub-movements of uniform duration and uniform relative accuracy as found by Crossman et al. [9]. The 

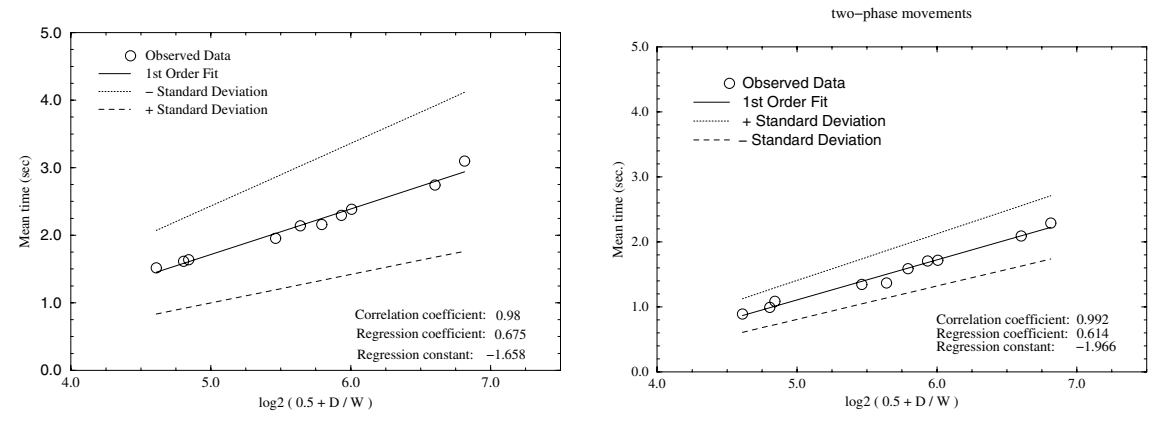

Fig. 2. Welford's variant of Fitts' law in the case of aggregate data (left) and considering only the ballistic and planning phases (right)

other class argues for the existence of two basic structural components; an initial impulse or ballistic component and a sequence of finer adjustments when approaching the target such as proposed by Welford [19]. Welford suggested based on these ideas that Fitts' index of difficulty should be reformulated into two terms in which the first term corresponds to an open-loop initial approach to the target, and the second term to a visually feedback controlled final alignment with the target. However, in experiments performed by Jagacinski et al. 9] with movements performed with a joystick between targets projected on a display of $38 \mathrm{~cm}$ by $28 \mathrm{~cm}$ the data collected were insufficient to establish conclusively whether the first sub-movement was regulated by an open- or closed-loop control. MacKenzie reports however that experiments have shown that movements that take less than $200 \mathrm{~ms}$ are ballistic and those with a duration over $200 \mathrm{~ms}$ are controlled by visual feedback [12] at page 118. This result has been obtained in the context of traditional Fitts' law experiments, so for amplitudes of at most $40 \mathrm{~cm}$.

Fig. 3] shows some examples of the velocity (top) and of the distance (bottom) profiles computed from trajectory and time-stamp data for different subjects and trials. From the graphs on the left, three phases are clearly identifiable during a movement:

- an initial planning phase characterised by a low velocity profile followed by

- a ballistic phase characterised by a high increase and subsequent decrease of the velocity profile followed by

- an adjustment phase characterised by a low velocity.

The graphs on the right show different examples of the velocity and distance profiles which have only two phases: a ballistic phase and an adjustment phase. In other words, in many trajectories the planning phase is not visible. The most likely explanation for this difference is the set-up of the experiment. In fact, participants have a view of the starting and target candidate circles for a trajectory on the board before they start operating the marker. That way, they might build 
a mental image of the board in advance and work on that image directly during the performance; that is, the planning phase is implicitly performed and the pointing task starts with the ballistic movement. Control over the position and size of the target is put in place at the end of the ballistic phase when adjustment is necessary. This requires a refresh of the mental image and the focusing on the image of the target. Consequently, the behaviour of participants doesn't show significant qualitative variations once the performance is started. It is interesting to note that each participant always adopts the same behaviour across multiple tasks (i.e. the presence/absence of the planning phase is invariant with respect to tasks for a subject). Apparently, a learning effect from previous knowledge of the position of the targets in the board is not appreciated. This may be due to the small number of tasks each subject is asked to perform together with the focusing on the current task only. However, two different strategies of operation are clearly revealed at this stage of our analysis. Further experiments are needed to study this phenomenon in a more controlled way. However, all subjects showed a trajectory with a ballistic phase followed by an adjustment phase when approaching the target.

If we correct the obtained data for the planning phase, i.e. we leave out the part of the trajectory that clearly concerns the planning phase, we obtain an even better fit of Welford's variant presented in Fig. 2 (right).

A further observation shown in Fig. 3 is that the velocity of the movement varies considerably as a function of the distance to the target. Moreover, velocities of more than 2 to 3 times as high as that of the average velocities based on Fitts' model can be observed. We discuss issues related to velocity in more detail in Section 3.4. In the next section we first look in more detail to the different phases of the pointing movements.

\subsection{Distance Covered and Time Spent in the Movement Phases}

The bar charts on the left of Fig. 4 show the mean percentage of the distance covered within the three distinguished phases of a movement, and the bar charts on the right show the mean percentage of time spent in those phases, for each of the indicated trials. It is evident that almost all of the distance was covered within the ballistic phase, while in the planning and the adjustment phases the distance covered is negligible. This occurred uniformly across all trials with minimal variations.

Considering the time spent to perform a complete movement, the variation across phases changes significantly. While the ballistic phase keeps taking most of the time, both planning and adjustment phases cannot be neglected.

The figure shows that the variation across target sizes of the percentage covered both in distance and in time during the planning phase is minimal. On the contrary, the adjustment phase duration depends on the size of the target both for distance and time: the bigger the target the shorter is the duration of the adjustment. 

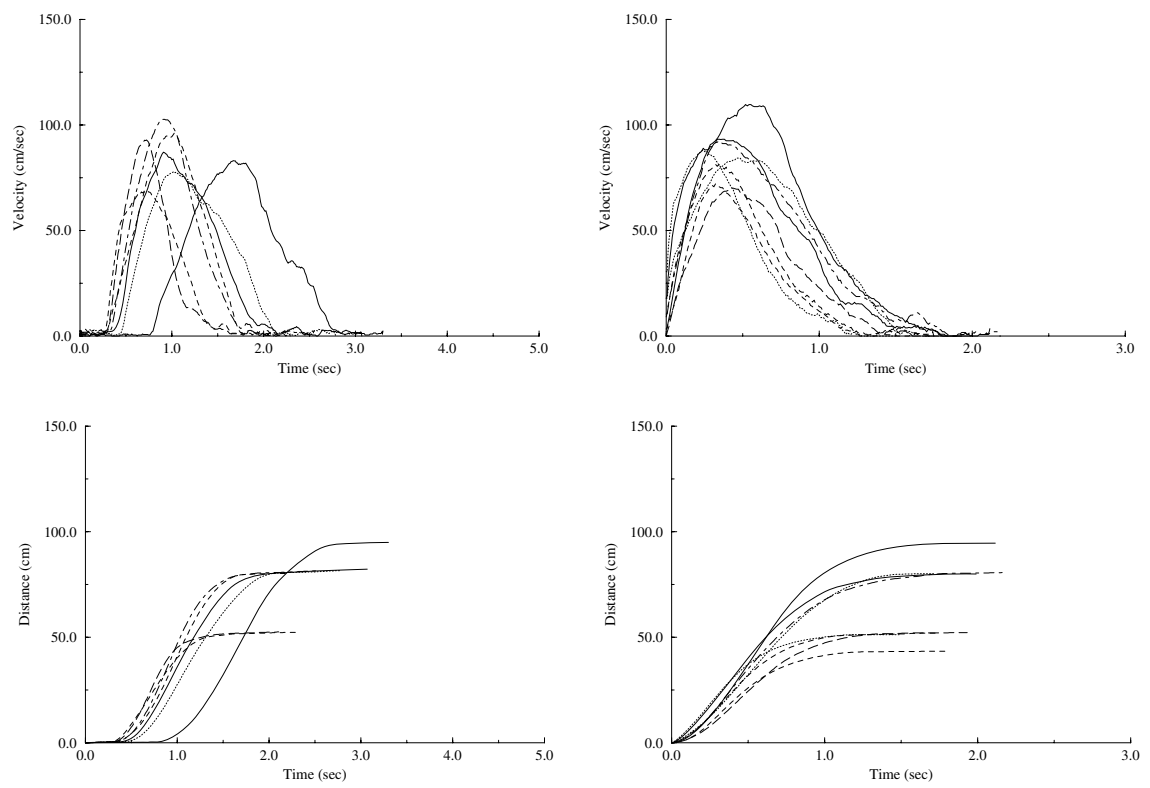

Fig. 3. Velocity (top) and distance (bottom) sample profiles with planning (left) and without planning (right) phases
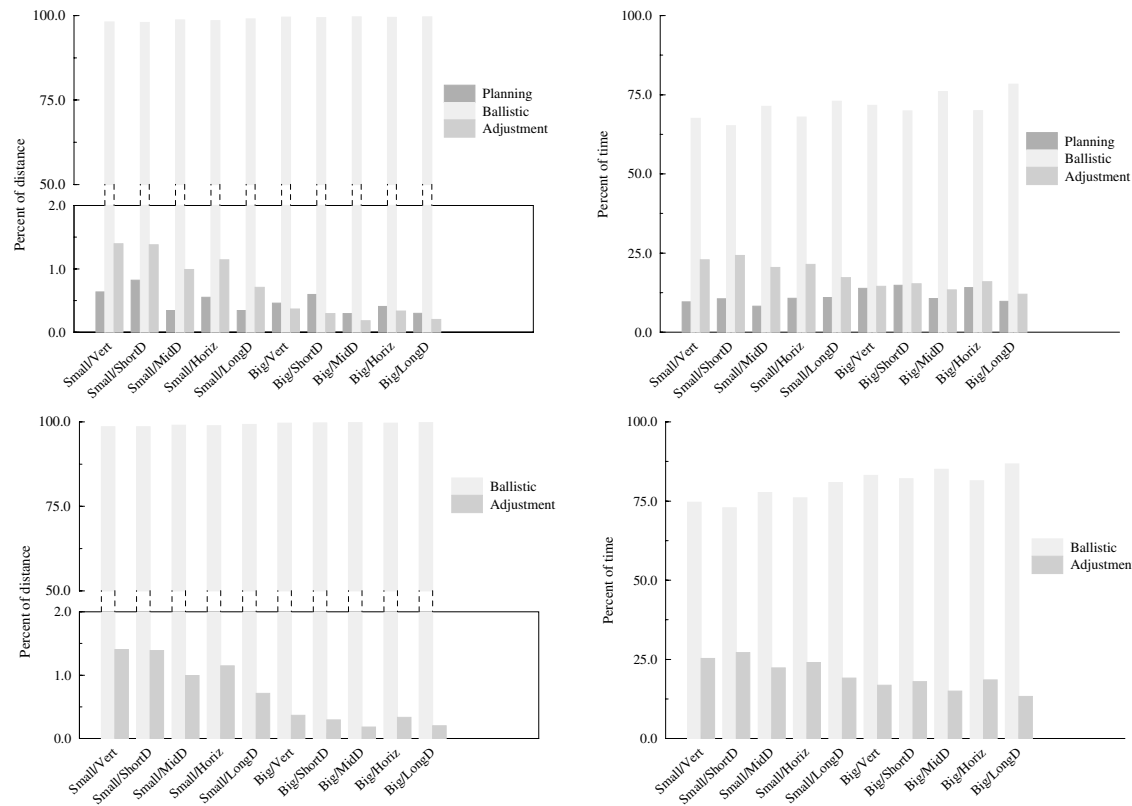

Fig. 4. Percent distribution of movements in distance (left) and time (right) per test 


\subsection{Observed Maximal Velocity}

Table 2 shows the maximal velocities that have been observed for each distance and each target width. It also shows the mean of the maximal velocities reached by the participants and the standard deviation. The highest velocity of 196.69 $\mathrm{cm} / \mathrm{s}$ has been reached for the target of $20 \mathrm{~mm}$. and the horizontal movement of $80 \mathrm{~cm}$ in a movement from point (1) to point (2) on the board. This is slightly less than the maximal velocity reported in [3] which was $200 \mathrm{~cm} / \mathrm{s}$. The latter however was obtained for a larger distance $(120 \mathrm{~cm}$.) and no clear target size. It can also be observed from this table that the highest mean maximal velocity is reached for the long diagonal and that this mean velocity decreases with the distance, with a minor exception for the short diagonal and the vertical movements for the small target.

Interestingly, from the same table we can also observe that the maximum velocity reached is not only depending on the distance that needs to be covered but also on the target size. Apparently, the ballistic movement is performed more cautiously and slower if a smaller target needs to be reached.

Table 2. Maximal observed velocity for each type of movement

\begin{tabular}{llrrr}
\hline $\begin{array}{l}\text { Target Movement } \\
(\mathrm{mm} .)\end{array}$ & $\begin{array}{r}\text { Type } \\
(\mathrm{cm} / \mathrm{s})\end{array}$ & $\begin{array}{r}\text { Velocity } \\
(\mathrm{cm} / \mathrm{s})\end{array}$ & \\
\hline 20 & Long diagonal & 178.47 & 95.29 & 33.12 \\
20 & Horizontal & 196.69 & 91.41 & 30.80 \\
20 & Mid diagonal & 162.69 & 79.18 & 31.01 \\
20 & Vertical & 149.58 & 76.18 & 30.84 \\
20 & Short diagonal & 171.07 & 69.56 & 26.99 \\
10 & Long diagonal & 109.66 & 67.35 & 18.81 \\
10 & Horizontal & 102.66 & 67.16 & 19.27 \\
10 & Mid diagonal & 99.94 & 54.25 & 17.66 \\
10 & Vertical & 95.68 & 48.95 & 15.11 \\
10 & Short diagonal & 92.79 & 49.22 & 14.85 \\
\hline
\end{tabular}

\subsection{Variability of Arrival Times}

Fitts' law studies typically do not address the distribution or variability of movement times but are aiming at the development of a valid model for the prediction of average movement times for different indices of difficulty and performance. Although Fitts' law has many important applications, there are situations in which the variability of the movement times is an important factor, such as in the case of direct interaction via computer vision techniques. It is well-known that human behaviour is quite variable, even in case of simple tasks, however, it is not completely random. Swain and Goodman [17, among others, observed for example that reaction times are rather well described by log-normal probability distributions. These are similar to normal distributions but skewed somewhat to the faster end of the distribution. 

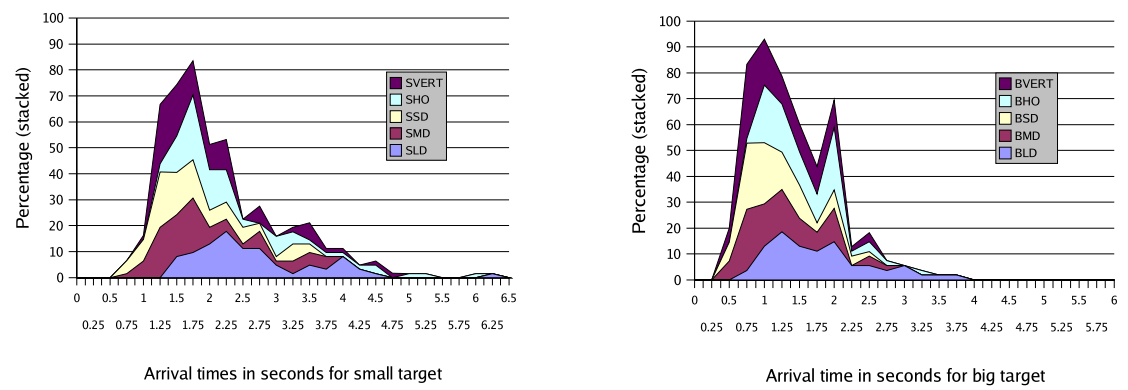

Fig. 5. Distribution of arrival time for small (left) and big (right) target for the different types of movement

In Fig. 5 the distribution of the arrival times for the two sizes of the target and the various types of movements are shown. On the horizontal axes the arrival time (in seconds) is shown. The vertical axes shows the percentage of trajectories that reached the target by that time in a stacked way. The numbers have been obtained by grouping the trajectories in slots of 0.25 seconds each. All trajectories have been renormalised by removing potential planning phases from the trajectories. Fig. 6] shows non-stacked distributions for the long diagonal trajectories for the small and the big target as an example.

It can be observed that the distributions are indeed skewed to the faster part of the distribution and resembles somewhat a log-normal distribution. However, in a number of cases a second, lower, peak can be observed toward the slower part of the distribution. This could be explained by the fact that movements of the same length, but in different directions have been grouped together. For example, moving from left to right might be much easier (and thus faster) for most people than moving from right to left. A further factor is that the number of movements considered in this study has been relatively limited. Further experimentation is needed to find out whether these distributions will be reproduced and would fit more closely to a log-normal distribution as these first data are suggesting.

\section{Discussion}

We have studied the pointing behaviour of adults performing simple pointing tasks on a white board. Such tasks involve movements over larger distances, and thus involving different limbs and body muscles than are usually considered in Fitts' law studies.

Although the study was limited in its set-up for what concerns the number of participants, and the number of trials that they were asked to perform, the use of an ultrasonic high-resolution movement capturing device provided interesting and detailed data on the structure of the movements and the variation of the velocity over each trajectory. Our results show a linear relationship between 


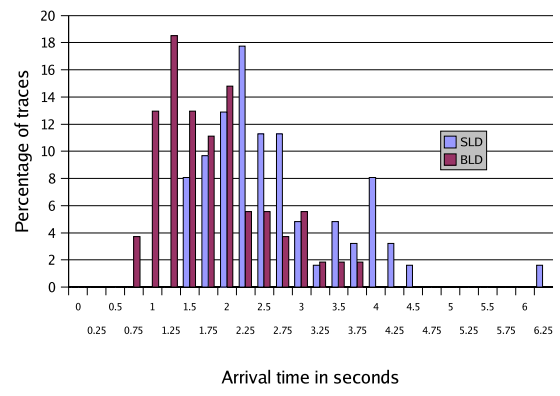

Fig. 6. Distribution of arrival time for small target, long diagonal (SLD) and big target, long diagonal (BLD)

movement time and the index of difficulty with a high correlation as in most Fitts' law studies for traditional pointing and tapping tasks. The main difference is that the regression coefficient was found to be much higher for pointing movements on the white board than in traditional Fitts' law experiments. This is in line with earlier findings that movements involving larger limbs are more sensitive to the index of difficulty. Furthermore, the obtained trajectories showed a clear number of phases in the structure of the movements. An initial planning phase, followed by a ballistic phase and an adjustment phase could be distinguished, although in some trajectories the planning phase was missing. This last aspect is most likely due to the way the experiments have been set-up. We plan to conduct further experiments in order to control this aspect as well as other aspects that seem relevant for the results, such as arm-length (reach), person's length and direction of movement.

The ballistic phase of the movements showed that velocities were reached that were significantly higher than the average velocity derived from the measured distance and movement times. Moreover, the velocity is clearly influenced by the size of the target. Furthermore, the data seems to suggest that the arrival times for each combination of distance and target follow a log-normal distribution. Further experimentation is needed to investigate this hypothesis in more detail.

For what concerns the design of vision based tracking techniques, the results of the experiments show that the velocity of the pointing movement varies considerably. Such knowledge could be used for improvement of the adaptive tracking techniques. For example, it could be investigated whether the initial part of the ballistic movement could be used to predict with a good accuracy in which direction and where the movement is heading.

Although limited in scope and number of participants, the current experiment shows nevertheless a number of interesting phenomena that would be worth to investigate further within the context of a larger experiment which we are currently carrying out. 


\section{References}

1. J. Accot and S. Zhai. Beyond Fitts' law: models for trajectory-based HCI tasks. In S. Pemberton, (Ed.), CHI-Conf. on Human Fact. in Comp. Sys.. ACM, 1997.

2. P. Barnard and J. May. Representing cognitive activity in complex tasks. Int. Journ. on Human-Computer Interaction, 14:92-158, 1999.

3. F. Bérard. Vision par ordinateur pour l'interaction homme-macine fortement couplée, 1999. Ph.D. thesis.

4. R. Cipolla and A. E. Pentland. Computer Vision for Human-Machine Interaction. Cambridge Univ. Press, Cambridge, UK, 1998.

5. G. Doherty, M. Massink, and G. Faconti. Reasoning about interactive systems with stochastic models. In C. Johnson, (Ed.), DSVIS . Springer, 2001. LNCS 2220.

6. G. Faconti, and M. Massink. Analysis of pointing tasks on a white board - Extended version. CNR-ISTI Technical report 2006-TR-24, CNR, 2006.

7. P. M. Fitts. The information capacity of the human motor system in controlling the amplitude of movement. Journ. of Exp. Psychology, 47:381-391, 1954.

8. Mimio interactive whiteboard, 2005. http://www.mimio.com.

9. R. J. Jagacinski, D. W. Repperger, M. S. Moran, S. L. Ward, and B. Glass. Fitts' law and the microstructure of rapid discrete movements. Journ. of Exp. Psychology: Human Perception and Performance, 6(2):309-320, 1980.

10. G. D. Langolf, D. B. Chaffin, and J. A. Foulke. An investigation of Fitts' law using a wide range of movement amplitudes. Journ. of Motor Behav., 8:113-128, 1976.

11. F. Letessier, J. Bérard. Visual tracking of bare fingers for interactive surfaces. In ACM Symposium UIST, Santa Fe, NM, USA, 2004.

12. I. S. MacKenzie. Fitt's law as a research and design tool in human-computer interaction. Int. Journ. of HCI, 7:91-139, 1992.

13. I. S. MacKenzie and R. Balakrishnan. Performance differences in the fingers, wrist, and forearm in computer input control. In S. Pemberton, (Ed.), ACM-CHI Conf. on Human Factors in Comp. Sys.. ACM Press, 1997.

14. I. S. MacKenzie and W. Soukoreff. Card, english, and burr (1978) - 25 years later. In Extended Abstracts of the ACM-CHI Conf. on Human Factors in Computing Systems, pages 760-761. ACM, 2003.

15. A. Murata. Extending effective target width in Fitts' law to a two-dimensional pointing task. Int. Journ. of Human-Computer Interaction, 11(2):137-152, 1999.

16. C. E. Shannon and W. Weaver. The mathematical theory of communication, 1949.

17. A. D. Swain and H. E. Guttmann. Handbook of human reliability analysis with emphasis on nuclear power plant applications - final report, 1983. Technical Report NRC FIN A 1188 NUREG/CR-1278 SAND80-0200. Prepared for Division of Facility Operations; Office of Nuclear Regulatory Research; Nuclear Regulatory Commission; Washington D.C. 20555.

18. A. T. Welford. The measurement of sensory-motor performance: survery and reappriasal of twelve years' progress. Ergonomics, 3:189-230, 1960.

19. A. T. Welford. Fundamentals of skill, 1968. 